\title{
A changing identity: a focus group study of the experiences of women diagnosed with secondary breast cancer
}

\author{
Ann M Baker, Ceri Phelps \\ Citation \\ Phelps C, Baker A (2018) A changing identity: a focus group study of the experiences of women diagnosed with secondary breast \\ cancer. Cancer Nursing Practice. doi: 10.7748/cnp.2018.e1549
}

Peer review

This article has been subject to external double-blind review and checked for plagiarism using automated software

\author{
Correspondence \\ ceri.phelps@uwtsd.ac.uk
}

\section{Conflict of interest}

None declared

Accepted

30 August 2018

\begin{abstract}
Aim: This paper reports the results of a study exploring the information and support needs of secondary breast cancer patients.

Method: A qualitative focus group study with 12 women with a diagnosis of secondary breast cancer in a UK hospital was carried out. Participants were aged between 29 and 70 years and had secondaries in bone, brain, liver and lung.

Findings: A thematic analysis revealed that the women felt unsupported by the care system compared to the level of support they received as primary breast cancer patients. Three overarching themes reflected an immediate sense of loss of formal support on diagnosis as a secondary breast cancer patient, a changed identity and associated self-stigma, and the emotional effects of living with the knowledge that their cancer was not curable.

Conclusion: Women living with secondary breast cancer have specific support needs that must be better recognised by healthcare providers. The extent to which secondary breast cancer nurses can meet these unmet needs should be evaluated.
\end{abstract}

\section{Author details}

Ceri Phelps, Director of psychology, University of Wales Trinity Saint David Swansea Campus, Psychology, Swansea, Wales; Ann M Baker, Secondary breast oncology clinical nurse specialist, Abertawe Bro Morgannwg University Health Board, South West Wales Cancer Centre, Singleton Hospital, Sketty, Wales

\section{Keywords}

\section{patient involvement, psychosocial; qualitative, secondary breast cancer}

\section{Background}

Secondary breast cancer (SBC), also known as metastatic (stage 4) breast cancer, occurs when primary breast cancer cells spread to other parts of the body, such as bone, liver, brain or lung, due to transmission through the blood or lymphatic system. It is not curable and results in prolonged cancer-related treatment and shortened life span. Recent estimates suggest around 35,000 people in the UK are living with SBC, with around five in every 100 people with breast cancer already having secondaries when their cancer is first diagnosed, and around 35 of every 100 people with primary breast cancer developing SBC within 10 years of their first diagnosis (Breast Cancer Now 2015).

With significant advances in the treatment and management of SBC that help to control the spread and relieve signs and symptoms, a diagnosis of SBC now reflects a chronic, potentially long-term condition. Consequently, women with SBC have complex and often unmet needs, report a higher level of psychological distress compared to what they experienced at primary diagnosis (Breast Cancer Care, Secondary Breast Cancer Taskforce 2007, 2008, Reed et al 2010, Breast Cancer Care (BCC) 2018) and higher levels of psychosocial morbidity (Cohen 2002, Coristine et al 2003). Feelings of uncertainty and lack of control, alongside stigma, 
clinical field (Hanson-Frost et al 2000, Mosher et al 2013, Krigel et al 2014). Over the last decade UK policy organisations and cancer charities have highlighted the need for improved specialist care and support for women with SBC. Early National Institute for Health and Care Excellence (NICE) guidelines (2004) emphasised the need to develop mechanisms to promote continuity of care for patients with advanced cancer and this agenda has continued to be driven forward by recent campaigns such as BCC's Secondary not second rate campaign. Encouragingly, the report produced from this campaign highlighted areas of good practice in service provision and support, which are a valuable starting point for addressing unmet needs, and include the provision of easily comprehensible written information covering clinical and support needs (BCC 2018). While UK cancer charities such as BCC and Macmillan provide a range of resources and information for patients with SBC, including written information, digital patient stories and support groups, women with the disease have unique characteristics and information and support needs that differ from those with early stage breast cancer and which are not being consistently met (Tucker et al 2017, BCC 2018, Macmillan 2018).

It is important that women with SBC have their emotional wellbeing regularly assessed and access to appropriate psychological support at diagnosis, at progression or when treatment changes (NICE 2009, BCC 2018). With the cancer clinical nurse specialist role shown to be valued by patients for the provision of emotional and information support (Ritz et al 2000, Halkett et al 2006,

Koutsopoulou et al 2010) much of the recent focus has been on access to a designated nurse specialist or keyworker with appropriate knowledge of the disease, treatments and support needs for patients with SBC, to act as an advocate and a link between the patient and the multidisciplinary team (MDT) (BCC 2018). However, according to the BCC (2018) report three-quarters of NHS trusts and health boards across the UK do not have enough specialist nursing care for those with SBC and women with the disease are often not signposted to appropriate support services.

Aim

This article explores the specific information and support needs of women with SBC through a qualitative lens. The aim of the study was to explore how women made sense of their experiences of being diagnosed with SBC and their associated information and support needs. The study formed part of the lead author's, a qualified breast cancer nurse, master's qualification in counselling practice. Using focus group methodology, the study objectives were:

» To explore women's experiences surrounding their SBC diagnosis.

» To gain an understanding of the emotional support and information needs of breast cancer patients since their secondary diagnosis.

» To discover whether women with SBC believe their needs have been met.

\section{Method}

The paper reports findings from two focus groups. The main researcher is a qualified nurse who has been working as a clinical nurse specialist in breast cancer care for over 20 years. Focus groups have long been recognised as an ideal way of obtaining rich narrative information about shared experiences of health and illness (Wilkinson 1998) and have been used as an aid to explore and plan future needs of women with SBC (BCC, Secondary Breast Cancer Taskforce 2008) and other cancer patient groups (Hughes and Phelps 2010, Harder et al 2017). Ethical approval for the study was obtained from the South West Wales Research Ethics Committee.

\section{Participants and recruitment strategy}

A purposive sample of women With SBC under the care of the oncology department at Singleton Hospital, Swansea were targeted for participation in the study between October 2013 and March 2015. Following ethical guidelines, the main researcher was not directly involved in the active recruitment of participants, including her own patients. For each focus group a poster advertising the study with an invitation to take part and contact numbers was placed on an information board in appropriate locations around the hospital. Interested participants had to contact the researcher to confirm their interest. When interest was expressed a participant information sheet and consent form was posted and once the consent forms were returned the researcher confirmed the date of the focus group.

Participants were eligible for inclusion if they had a definite diagnosis of metastatic breast cancer made by either cytology or tissue biopsy or by radiological investigation. Women with bone, brain, liver and lung metastases were also eligible. Two core exclusion criteria were if the woman became an oncological emergency warranting urgent hospital admission and those considered by the clinical team as too psychologically vulnerable to participate.

Recruitment of the first six women was in October 2013 and the focus group took place in December 2013. The second focus group comprising another six women took place 15 months later, following difficulties recruiting women during this period due to their poor health or death. Across both groups the women had secondaries in bone, brain, liver and lung and their age ranged from 29 to 70 years. 
Importantly, within the context of the study, by the time the second group took place a support group had been established for SBC patients driven by those who had taken part in the first group. At the time of writing nine of the participants were still part of the support group while three have died.

\section{Data collection and analysis}

Each focus group was held in a comfortable setting in the hospital with soft, comfortable seating and easy access to amenities, and lasted for 90 minutes. The main researcher (AB) supported the group using a semi-structured topic guide with the help of a comoderator (CP). The topic guide explored the women's preferences in relation to their need for information and support since their SBC diagnosis, the type of support they believe they would have benefitted from and the ongoing support they believe would make a difference. Discussions from the focus groups were transcribed verbatim and the transcripts were anonymised using pseudonyms. The data were analysed using thematic analysis following Braun and Clarke’s (2006) framework due its flexible approach that enables researchers to identify commonly occurring themes without being overly influenced by a strong theoretical stance.

\section{Findings}

Thematic analysis of the focus group data revealed three overarching superordinate themes with underpinning subordinate themes (Table 1) which are discussed in depth below. Where differences appeared to exist between the first (G1) and second (G2) focus group as a consequence of the support group these are interpreted below within this context. All names are pseudonyms.

\section{Table 1. Superordinate and subordinate themes resulting from thematic analysis}

\begin{tabular}{|l|l|l|}
\hline $\begin{array}{l}\text { Superordinate theme 1: Loss of } \\
\text { support }\end{array}$ & $\begin{array}{l}\text { Superordinate theme 2: Changing } \\
\text { identity }\end{array}$ & $\begin{array}{l}\text { Superordinate theme 3: } \\
\text { Living with no cure }\end{array}$ \\
\hline Sense of abandonment & Self-stigma & An uncertain future \\
\hline Needing time to reflect & $\begin{array}{l}\text { A common identity through shared } \\
\text { experience }\end{array}$ & Resilience \\
\hline $\begin{array}{l}\text { Perceived reluctance to explore the } \\
\text { death question }\end{array}$ & & $\begin{array}{l}\text { Reluctance to seek } \\
\text { psychosocial support }\end{array}$ \\
\hline A missing link & & \\
\hline
\end{tabular}

\section{Superordinate theme 1: Loss of support}

One of the main questions the participants were asked was to think about the type of support they were offered at the time of their SBC diagnosis. A strong overarching theme reoccurred across both focus groups as the women reflected on this time. All participants recalled readily the moment they were informed that their cancer was non-curable and many of their reflections involved comparison with their primary breast cancer diagnosis.

\section{Sense of abandonment}

There was a strong and pervasive sense of loss of support and an almost immediate sense of abandonment at the moment of the secondary diagnosis. For example, one woman said: 'I was only finding out that I had secondaries this year and I feel totally abandoned by you know any support whatsoever since I got referred to oncology' (Mary G1) while another said: 'I had loads of support and everything, but then when we were given to oncology I feel I was treated like a statistic not as a person, and you do feel lost’ (Susan G1).

A similar sentiment was echoed by participants in the second focus group: 'The doctors come in and they just go "du dudu, this is what you have got” and they, they go... and then the nurse or whoever is left there...' (Lucie G2). Another participant said she was '...sent out of the room a quivering wreck' (Robyn G2).

During discussions about their experiences the women identified with similar feelings voiced by others in the group and in both focus groups the effect of this sharing of emotion appeared powerful leading to some tears and comfort from fellow participants: 'I feel the same. Once you find out they leave you. You do not know nothing about it. You gets told and then you just walk out of there and you think well where do I go from here?' (Tess G1). At this point the participant became tearful.

\section{Needing time to reflect}

As the discussion went on it became evident that some women believed they had not been given sufficient time or information to fully understand their diagnosis: 'On reflection, it would have been nice if they said this is really hard for you to take in, here's a number for when you feel a bit calmer. Then they can explain anything' (Robyn Group 2).

It was also clear that this need for understanding and seeking answers continued after the initial diagnostic consultation: 'I mean I do not think any of us will ever forget the day you told that initial conversation and you walk out of the room and you are in there and you think right keep your head straight ask the questions, and I will ask the questions and then you go outside and you sit down and you just shocked, and of course that shock wave may come over you within a couple of hours, oh I want to ask this, I want to ask that' 
(Hannah G1). Another participant said: 'I felt, I do not know perhaps, if I reflect a bit, perhaps I just blanked out and I did not take it in, I just switched off. It was the most unbelievable day, I just felt I was left to walk out and find my own way. What shall I do? I came home and rang (a cancer charity), they did not tell me anything nice. So, I will not be ringing them again (she laughs)' (Robyn G2).

\section{Perceived reluctance to explore the death question}

Across the focus groups, as the discussions unfolded and the women started to share their experiences, they expressed feelings of frustration, confusion and resentment about what was often perceived as unwillingness on the part of medical professionals to discuss the details of their diagnosis, particularly in relation to questions about the non-curable nature of the diagnosis: 'When they have said you have got cancer again and I just went and I said “am I going to die?” and I do not know why I said it, but they said "well I do not know”! and I will never forget, never ever and I do not want to see them' (Edna G1.)

For some the frustration appeared to stem from a sense that their consultant or oncologist was fixated on the fact that their cancer was incurable and therefore seemed reluctant to engage in in-depth discussion of wider questions. For example, one woman recalled: 'He says “you do realise you cannot be cured, you do realise you cannot be cured?”' (Susan G1) and continued 'I find that really annoying when I go to my oncologist and say, well I would say in, what, a 20 minutes consultation, that if I do not hear that word (incurable) three times'.

Similarly, Edna voiced frustrations when she 'asked about something and they sort of fob it off a little bit, because I asked about a trial and the oncologist “ooh, I had not heard about this”, and I says “well I thought you would have heard about this”” (Edna G1).

Due to these earlier frustrations some of the women appeared to have developed strategies for future consultations to ensure they could ask questions they believed were important. For example, Susan said she ensured she went prepared to every consultation to get answers to her questions: 'I ask many questions when I go in to see my oncologist. I take a list in and he does, he does dread seeing me because you can see it in his face the minute I walk in through the door’' (Susan G1).

\section{A missing link}

While all the women in both groups agreed with the shared sentiment of a change in the level of care following the SBC diagnosis, a few talked about have receiving excellent levels of care throughout their cancer pathway. For these women there appeared to be a much shorter time between the diagnosis of primary and SBC and a specific individual had helped to create a more seamless transition to their new status: 'So I was under the chemo unit anyway, but xxxx was fantastic, but I was on tablets so I could see her she put me in touch with Macmillan so I had loads of support from Macmillan’ (Hannah G1).

Participants appeared to feel there was a missing link between them and oncology following their secondary diagnosis: 'That is why you need somebody in between' (Edna G1).

They also talked about how they understood that the oncologist had many patients to see and limited time which appeared to create a barrier to them actively seeking further advice or support: 'It would be nice if somebody could be in that middle to help him and help us because then you think because I said I cannot phone up, I said, because they think I am being a nuisance' (Edna G1).

A number of participants asked why there were primary breast cancer nurses at their hospital and no allocated nurse for SBC: 'If $30 \%$ of women get secondary breast cancer why is not one of those nurses designated as a secondary breast cancer nurse?' (Mary G1); 'We need a secondary breast cancer nurse!' (Angharad G2).

\section{Superordinate theme 2: Changing identity}

The diagnosis of SBC appeared to have a fundamental effect on all participants' self of sense and how they felt perceived by others. Some shared stories that revealed a change in self identity from being a patient with primary breast cancer to one with an SBC diagnosis. This change in identity often appeared to be in stark contrast to how they felt on their initial diagnosis months or years earlier and explicitly linked to the label 'incurable'.

\section{Self-stigma}

Mary (G1) explained: 'I mean my primary care it was fantastic - was amazing - and the care I got was amazing but I just think 13 years, you get secondary breast cancer and I almost feel like a bit of a second class citizen really, you know, how are you meant to feel?'

One participant who had regularly attended events held by a cancer charity said she did not feel she used it much since her SBC diagnosis and talked about how she felt different in the view of the people she came into contact with who had primary breast cancer. She now felt that she had a different identify to those with primary breast cancer: 'But sometimes if I am feeling a bit a low then this is not really, because if someone starts asking me then I am going to end up being upset, which is not going to help them you know, if 
they have got primary cancer, not secondary cancer it is quite possible they will get better so they don't need to see me crying and going oh yeah but sorry I am not going to get better particularly if it’s breast cancer, as so many people do get better' (Hannah G1).

Findings from the analysis were alarming; the lack of support meant the women felt isolated and stranded and this made them feel different. However, one participant who had been attending the chemotherapy day unit when she recurred stated: 'I must admit I have to say I must be the odd one in the group whether it is because my head did not really stop between finding that I had cancer, and as soon as my meds stopped my second cancer came back straightaway’ (Hannah G1).

Another participant spoke eloquently about the lack of continuity and poor communication and her fight to be noticed as a whole person: 'So I feel that nobody is treating me holistically because you cannot separate parts of my body' (Angharad G1).

\section{A common identity through shared experience}

For these women participating in the focus groups appeared to evoke a sense of a shared experience and a common identity, and this was particularly noticeable in the first group where participants shared their frustrations, tears and pain in a frank and honest manner. In the first group, during the introductions, all the women said how pleased they felt to be in a group with others with SBC: 'This is a group that is long overdue and I am really keen to support' (Angharad G1); 'I was moved then to oncology. I feel totally abandoned and unsupported and yeah so I think something like this group is fantastic for me. Yeah' (Mary G1).

Following this initial focus group a SBC support group was established by the participants supported by AB. The support group had met on a number of occasions before the second focus group and provided a much-needed mechanism for sharing experiences which the women had not had before: 'I feel I belong somewhere. I fitted in’ (Amy G2).

Participants in the second focus group talked about the comfort and reassurance they gained from attending the support group and the effect it had on their self-stigma and perceptions:

» Josie: 'The first time I came to the group it was a big comfort in that, Mary, I remember the name because it struck me that everybody looked normal and you think that you are going to be walking around you know with Dr Death over you. But everyone looked normal so that was a comfort and it was a comfort when Mary said "oh well I was climbing the alps and I rely on Pinot Grigio and After Eights” were her exact words and I was thinking well there is hope then. There is hope, where I had felt written off, and then I went home and I had pizza and wine 'cos I had been frightened to drink, frightened to have rubbish... (long sigh) it gave me hope. The group the first time gave me hope, that I did not have before, it lifted me.'

» Nerys: 'It is not a morbid group is it?'

» Amy: 'No it’s fab.'

» Josie: 'It is funny.'

» Serena: 'I thought everyone is going to be iller than me, disabled on walking sticks and they are not. Yes, so I took a few months to get here, two months, because my nurse had said, and Maggie’s had said, but I said ooh I am not ready for that yet. So, I did not come you know, on my diagnosis, I was just going to deal with it really.'

Finally, some participants talked about how well-meaning comments from family and friends were often hard to accept: 'I always say "you walk in my shoes” and then, but you cannot say to these people... they have not got cancer’ (Edna G1). For example, one of the focus group discussions centred on being told to ‘be positive' which evoked a range of opinions: 'I just say yes I am positive, positively pissed off!' (Mary G1) (group laughter).

\section{Superordinate theme 3: Living with no cure}

This theme reflects the wider emotional journey of living with the knowledge that ultimately these women are facing an early death. However, while early death was considered inevitable there was much emotional uncertainty about preparing for the event, a sense of resilience and determination to live for as long as they could and what appeared to be an emerging reluctance to proactively seek out and engage with a variety of psychosocial support options.

\section{An uncertain future}

There was a common thread that weaved through the findings in relation to the co-occurrence of guilt and fear in relation to what the future holds for their families and loved ones and the effect of them not 'being there': 'You are sitting there and you get all these dark thoughts, and you start thinking Oh God, in my case, it was what if I am not there to see my kids finish school, or what if I am not there to see them when they need me’ (Hannah G1). Robyn (G2) talked about the difficulties of putting on a brave face for family: 'But I do find the emotional side was the hardest for me to cope with, my Christmas was the most miserable ever despite trying to put on a brave face and let's carry on.' 
One of the women who validated the themes emailed a further explanation: 'Guilt, effect on kids/not here to see them finish school, or be there if they need me... I feel this way about my parents, who will care for them? Not wanting them to feel the pain of losing a child, maybe I am being a little immature with my feelings, but it is real all the same, but I am sure there must be other ladies out there in the same situation as me. I am sure the feelings are the same when thinking about husbands and partners, how will they cope?'

(Susan G1).

\section{Resilience}

Despite the emotional effect of hearing the traumatic news that they have SBC the women showed great resilience to help them get some control to cope and function day to day. Hannah (G1) explained that: 'Everyone tells me I am not curable, I am only not curable when I get to that point when they say actually there is nothing else we can do for you.' She went on to explain that: 'They say it is not curable and I feel that the way it has been put its almost to keep my foot on the floor so that I realise that I have got to keep fighting, and I keep saying well you know, I am fine, I am going for the 10 year, my joke is I am going for the 10 year plan.'

Some participants talked about the ongoing sense of challenge and refusal to be defined by their diagnosis: 'Well I will not let it identify me. I am far more, I have got far more to say about myself, even though I feel as if I have got myself into a bit of a mess' (Angharad G1). Similarly, Susan (G1) stated that: '’Cos I challenge it 'cos I am like yourself, it is not cured until the day I die, until that point’, while Hannah (G1) said: ‘This what I keep drumming into the kids all the time, I keep saying I have not given up so you must not give up.'

Throughout the discussions it became clear that many of the women use humour as a coping strategy with their families: 'It is just I tend to, I just say to the kids do you know I have made up my mind I am going to get better so that is what I am doing and that is, you know, I said I just cannot be doing with this being ill stuff that it is a waste of time and that is how we joke it out so it is you know' (Hannah G1).

During the discussions themselves, although the laughter was mixed with tears at times, the camaraderie and connection of both newly formed groups appeared almost instant. Nerys (G2) said: 'I want to know, I want to know how much time I have got to tidy my bloody house, before someone says “why has mother kept this for” like I did with mine’ (group laughter).

\section{Limited engagement with psychosocial support}

Although there was a clear perception across both groups that their emotional wellbeing was not regularly, if ever, assessed through the care pathway, a powerful finding, particularly in the first group, was that the women struggled to articulate the specific types of support they has been offered as they felt the support they had received was minimal or non-existent. Although some were aware of services offered by local and national cancer charities they felt the onus was on them to make contact and that was not always easy for some of them, while signposting to additional support organisations and cancer charities often came from nonformal networks such as family and friends: ‘It was my daughter-in-law that said “why do not you go to Maggie’s?” and I went, what is Maggie’s? why was not I told that?' (Mary G1).

Susan (G1) expressed a sentiment endorsed by other participants which was a reluctance to make contact proactively with other sources of support even when they had been made aware of it: 'I was just left thinking oh my god have I got cancer in my back because I have been in pain since well for six months, not knowing I had breast cancer um, and that I could have done with somebody to ring then even though yes I would have had my booklet, which said ring Macmillan or ring the Breast Org, but I am the type of person I find it hard to make that first call’.

In the second focus group, the fact that patients were being signposted to the newly established support group was providing a mechanism to share information about sources of support as well as providing important social support: 'My nurse came in with some reassurance and then I found out the secondary group was going. I did not even know what secondary meant' (Robyn G2).

There appeared to be varying levels of awareness about the availability of counselling services. Three of the women had received some counselling and felt they had benefitted from it, allowing them to explore their feelings and identify coping strategies: 'I think one-to-one counselling session is invaluable' (Mary G1).

However, not all women had been offered counselling and one explained: 'She was too busy fighting on an academic information level to be concerned for her emotional needs' (Angharad G1).

There was some acknowledgement that there was limited signposting to these services from clinicians themselves: 'Although it seems very much, I love my oncologist I love him, and other doctors have been very fair at this stage of the treatment, but no one asks you how you are ‘cos they cannot offer you a counselling service. They have not got time, it takes long enough’ (Josie G2). 


\section{Disc ussion}

The findings indicate that women with SBC are continuing to report specific information and support needs that are not met consistently by oncology care pathways. An important finding is that their experiences of being SBC patients were often in stark contrast to those as primary breast cancer patients, with the overarching themes reflecting a powerful narrative of an almost immediate sense of loss of formal support, the perception of changed identity and associated self-stigma and the emotional effects of living with the knowledge that their cancer was incurable.

Health professionals are perceived as the gatekeepers of information, yet there is a distressing and frustrating divide between the way in which clinicians convey information to patients, the nature of this dialogue and patients' needs to understand fully the clinical information provided. Central to reducing the many barriers perceived by women with SBC, to enable greater engagement with their own future, appears to be a trusted gatekeeper between the various clinical cancer services directly involved in their care and other appropriate support organisations. The findings support previous research that indicated that access to appropriate psychosocial and information support is an important mechanism for empowering patients with SBC and improving quality of life (Pender 2002, Reed et al 2012, Begley et al 2014). Participants in this study valued clinical nurse specialists who are regarded as a vital source of knowledge about the disease and treatments and an important advocate of their clinical and psychosocial support needs, thus acting as a crucial link between the women and the multidisciplinary team.

Findings highlight the importance of regular holistic needs assessments at diagnosis and beyond and indicate the importance of a suitably skilled and experienced person to take on an advocacy role beyond simple signposting. This suggests that alternative roles, such as support workers who focus solely on providing emotional rather than information support, are unlikely to address these women's unmet needs. It is concerning, therefore, that in Wales the Cancer Delivery Plan (Welsh Government 2016) makes little consideration of the needs of women with this cancer diagnosis, and that the key worker role, as currently construed, does not factor in how these women might have concerns different from those with a primary diagnosis.

As a consequence of the study the lead author is working as Wales' first dedicated specialist SBC nurse enabling patients to access specialist support and nursing care in line with existing recommendations, for example BCC (2018). While not a specific objective of the study, the insight into the demands and expectations of this SBC nurse specialist role suggests that such roles need to be supported appropriately by service providers to remain sustainable. With recognition of the ambiguity about the potential scope of breast cancer nurse specialist roles (Jones et al 2010), the BCC (2018) acknowledges the need for appropriate training in specialist skills and knowledge. However, there may also need to be recognition of the need to provide appropriate emotional support, whether through training, counselling skills or increased clinical supervision, for those working in SBC care. Therefore, while there is undoubtedly evidence that such a role is valued by patients, there is also a need for systematic evaluation of effects of these specialised roles on the lived experience of those with SBC and on specialist SBC nurses.

While the role of specialist SBC nurses appears crucial in supporting a more holistic care pathway and patient experience, the findings from this study also indicate that, in line with the wider cancer literature (Moore et al 2013, Tucker et al 2017), there is a need to explore opportunities to improve communication skills between patients with SBC and other oncology professionals, including consultants. Much of the anxiety and negative experiences reported in the study appear to stem from frustrating conversations with a range of oncology professionals who, to the women involved, did not seem willing to engage in difficult conversations about their diagnosis and living with uncertainty. Additionally, the self-stigma that some of the participants reported appears to act as a barrier to pushing for more clinical or informal support. While the link between perceptions of stigma and reluctance to seek help has been evidenced in other fields such as lung cancer (Carter-Harris 2015) this is an important finding in relation to SBC which must be addressed by the clinical, psychosocial care and research disciplines through appropriate education and intervention.

The findings suggest, therefore, that oncology professionals may need further training to develop effective communication strategies that help reduce a perception of stigma among their patients and enable them to feel they can raise issues related to their SBC legitimately at the time of diagnosis and beyond. With recognition that the longer a person lives with breast cancer the less support they may receive (BCC 2018), this study highlights the need for ensuring continuity of clinical and psychosocial support over time and not just in the immediate aftermath of the secondary diagnosis. Initiatives such as the BCC Secondary Toolkit (2012) provide easy access training resources for nurses working in the SBC, however there also appears to be a need for additional resources or training for other oncology professionals. 
This study highlights the value of focus group methodology in helping to assist with decision making and planning for implementation of policy and care packages (Krueger and Casey 2000). The fact that the opportunity to take part in this study appeared to provide a platform for the participants to establish their own monthly support group illustrates the value of peer support initiatives in providing important support and reducing feelings of isolation and self-stigma. The Breast of Friends SBC Support Group still meets monthly with up to 20 women attending events and supporting each other at funerals. The group was also instrumental in supporting the lead author to organise an SBC awareness conference in October 2017 attended by over 100 healthcare professionals and patients. That these women felt empowered to establish such a group, with ongoing support from their SBC nurse, further highlights the value of patient engagement and the 'patient voice' in understanding perceptions of 'good care' and shaping the future delivery of services (Begley et al 2014, BCC 2018). Indeed, this must be pushed one step further by involving patients in the codesign of SBC services and ensuring that specialist SBC nurses believe they can shape these unique services with their patients.

Our ongoing research will seek to identify, develop and pilot a brief communication skills training intervention for healthcare professionals, coproduced by women with SBC, and focused on dealing with uncertainty and unanswered questions about a diagnosis of SBC.

\section{Limitations}

The small-scale nature of this research is recognised as a limitation of the current study, with the views of a relatively small number of women not necessarily representative of all experiences of women with secondary breast cancer. We also recognise that the study participants came from one local health board which again may not be reflective of the experiences of women under the care of other health boards/NHS trusts. Within our sample, however, we were able to achieve an in-depth understanding of the experiences of women across a range of ages and time since diagnosis and believe that this rich data can help illuminate many of the ongoing challenges faced by women with a diagnosis of secondary breast cancer across the UK.

\section{Conclusion}

The results of this focus group study indicate that women with SBC still have unmet information and psychological support needs and there appears to be a missing link in the support offered compared to their experiences as primary breast cancer patients. Women with SBC require a more holistic care delivery and improved communication between them and their breast multidisciplinary team. In evaluating the effect of such initiatives or service delivery changes it is important that patients' voices are not forgotten to truly understand the ways in which we can best empower these women to face the many challenges of an uncertain future.

\section{Implications for practice}

» Women with SBC need to be assessed regularly for their emotional wellbeing and signposted appropriately to additional psychosocial support at diagnosis and as the disease progresses.

» Oncology healthcare professionals must be made more aware of effective communication strategies to reduce self-stigma among their patients and with regard to difficult conversations about a diagnosis of SBC.

» The role of dedicated SBC nurse offers an important opportunity to breach the gaps in information provision and support. The extent to which this role needs to be supported through appropriate clinical and emotional supervision is important to address.

» The power of the patient voice in driving change and shaping services needs to be better recognised and embedded in the codesign and co-development of future SBC services. Engaging healthcare professionals and patients in the codesign and coproduction of resources that will improve conversations and information giving in relation to a SBC diagnosis may help improve patients’ experiences and quality of life.

\section{References}

Begley A, Pritchard Jones K, Biriotti M et al (2014) Listening to patients with cancer: using a literary-based research method to understand patient-focused care. BMJ Open. 4,10 , e005550.

Braun V, Clarke V (2006) Using thematic analysis in psychology. Qualitative Research in Psychology. 3, 2, 77-101.

Breast Cancer Care (2012) Ensuring Nursing Provision for People with Metastatic Breast Cancer A Toolkit for Healthcare Professionals. www.breastcancercare.org.uk/sites/default/files/files/sbc nursing toolkit 2012 0.pdf (Last accessed: 15 November 2018.)

Breast Cancer Care (2018) Secondary not Second Rate. www.breastcancercare.org.uk/sites/default/files/cool085_the_case_for_change_report_final.pdf (Last accessed: 4 September 2018.) 
Breast Cancer Care, Secondary Breast Cancer Taskforce (2007) Access to a Key Worker for All Secondary Breast Cancer Patients: Setting the Scene. Breast Cancer Care, London.

Breast Cancer Care, Secondary Breast Cancer Taskforce (2008) Improving the Care of People with Secondary Breast Cancer: The Final Report. Breast Cancer Care, London.

Breast Cancer Now (2015) www.breastcancernow.org/about-breast-cancer/want-to-know-about-secondary-breast-cancer/how-common-is-secondary-breast-cancer

Carter-Harris L (2015) Lung cancer stigma as a barrier to medical help-seeking behavior: practice implications. Journal of the American Association of Nurse Practitioners. 27, 5, 240-245

Cohen M (2002) Coping and emotional distress in primary and recurrent breast cancer patients. Journal of Clinical Psychology in Medical Settings. 9, 3, 245-251

Coristine M, Crooks D, Grunfeld E et al (2003) Caregiving for women with advanced breast cancer. Psycho-oncology. 12, 7, 709-719.

Halkett G, Arbon P, Scutter S et al (2006) The role of the breast care nurse during treatment for early breast cancer: the patient's perspective. Contemporary Nurse. 23 $1,46-57$.

Hanson-Frost M, Suman V, Rummans T et al (2000) Physical, psychological and social well-being of women with breast cancer: the influence of disease phase. Psycho-oncology. 9, 3, 221-231.

Harder $\mathrm{H}$, Holroyd P, Burkinshaw L et al (2017) A user-centred approach to developing bWell, a mobile app for arm and shoulder exercises after breast cancer treatment. Journal of Cancer Survivorship : Research and Practice. 11, 6, 732-742.

Hughes L, Phelps C (2010) "The bigger the network the bigger the bowl of cherries.Maybe there's a cherry for every little problem you might come across.": exploring the acceptability of, and preferences for, an ongoing support network for known BRCA 1 and BRCA 2 mutation carriers. Journal of Genetic Counseling. 19, 5, 487-496.

Jones L, Leach L, Chambers S et al (2010) Scope of practice of the breast care nurse: a comparison of health professional perspectives. European Journal of Oncology Nursing : The Official Journal of European Oncology Nursing Society. 14, 4, 322-327.

Koutsopoulou S, Papathanassoglou E, Katapodi M et al (2010) A critical review of the evidence for nurses as information providers to cancer patients. Journal of Clinical Nursing. 19, 5-6, 749-765

Krigel S, Myers J, Befort C et al (2014) 'Cancer changes everything!' Exploring the lived experiences of women with metastatic breast cancer. International Journal of Palliative Nursing. 20, 7, 334-342.

Krueger R Casey M (2000) Focus Groups: A Practical Guide for Applied Research. Sage, London.

Macmillan (2018) www.macmillan.org.uk/information-and-support/breast-cancer-secondary

Moore P, Rivera Mercado S, Grez Artigues M et al (2013) Communication skills training for healthcare professionals working with people who have cancer. Cochrane Database of Systematic Reviews. 3, Art. No.: CD003751.

Mosher C, Johnson C, Dickler M et al (2013) Living with metastatic breast cancer: a qualitative analysis of physical, psychological, and social sequelae. Breast Journal. $19,3,285-292$

National Institute for Health and Care Excellence (2004) Improving Supportive and Palliative Care for Adults with Cancer. National Institute for Health and Care Excellence, London.

National Institute for Health and Care Excellence (2009) Advanced Breast Cancer: Diagnosis and Treatment Clinical Guideline. National Institute for Health and Care Excellence, London.

Pender S (2002) Patient empowerment through information. Cancer Nursing Practice. 1, 6, 32-38.

Reed E, Scanlon K, Fenlon D (2010) A survey of provision of breast care nursing of patients with metastatic breast cancer: implications for the role. European Journal of Cancer Care. 19, 5, 575-580

Reed E, Wheeler D, Scanlon K (2012) Spotlight on secondary breast cancer: a policy initiative to influence the care of women with metastatic breast cancer. Breast Cancer Management. 1, 3, 201-207.

Ritz L, Nissen M, Swenson K et al (2000) Effects of advanced nursing care on quality of life and cost outcomes of women diagnosed with breast cancer. Oncology Nursing Forum. 27, 6, 923-932.

Tucker C, Martin M, Jones R (2017) Health information needs, source preferences and engagement behaviours of women with metastatic breast cancer across the care continuum: protocol for a scoping review. BMJ Open. 7, 2, e013619.

Wilkinson S (1998) Focus groups in health research. exploring the meanings of health and illness. Journal of Health Psychology. 3, 3, 329-348.

Welsh Government (2016) Wales Cancer Delivery Plan. Welsh Government, Cardiff. 